\title{
Synthesis of Erythromycin Derivatives via the Olefin
}

\section{Cross-Metathesis Reaction}

\author{
Margaret C. Hsu*, Adam J. Junia , Anthony R. Haight, Weijiang Zhang ${ }^{*}$
}

Process Chemistry Research and Development, Global Pharmaceutical R\&D, Abbott Laboratories,

1401 Sheridan Rd., North Chicago, Illinois 60064, USA.

Current address: Department of Chemistry, 907 Washington Street, Northwestern University, Evanston, Illinois 60202, USA.

${ }^{\ddagger}$ Current address: Theravance Inc., 901 Gateway Blvd., South San Francisco, California 94080, USA. 
Table of Contents:

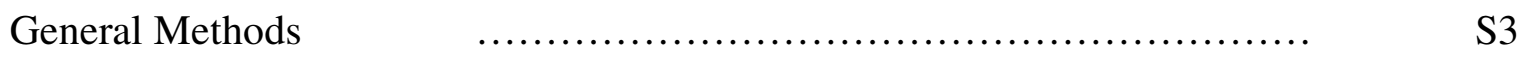

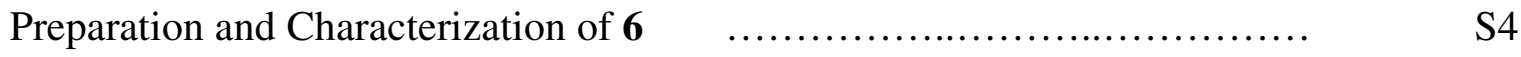

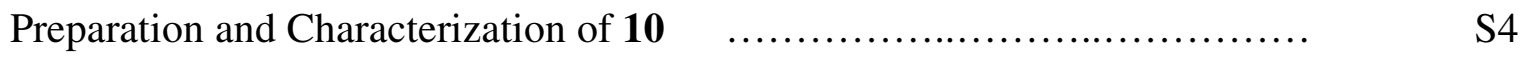

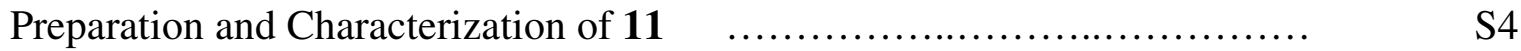

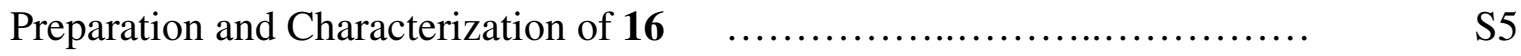

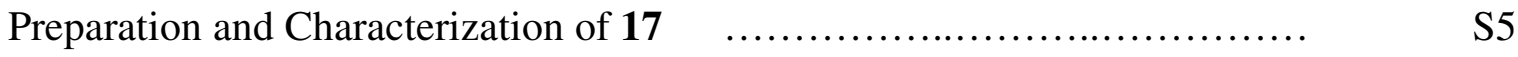

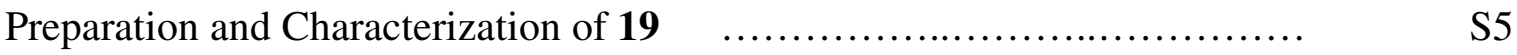

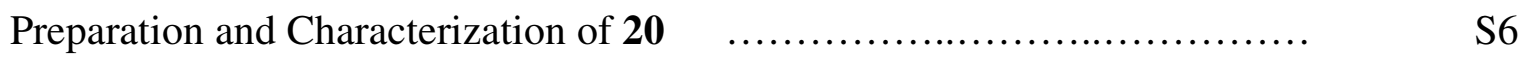

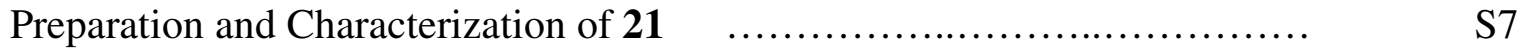

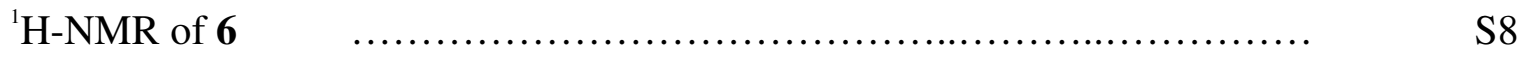

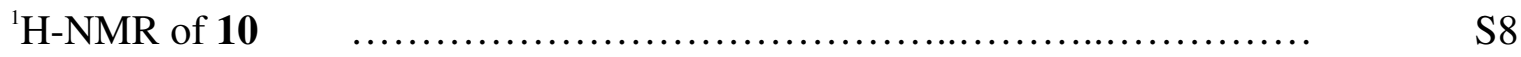

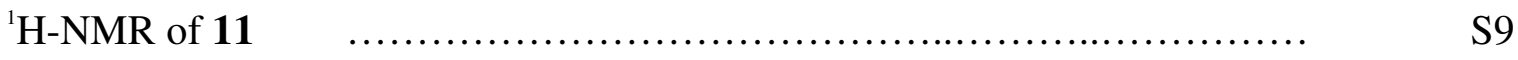

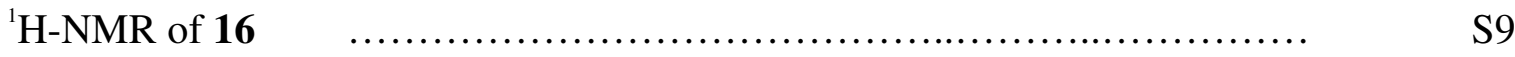

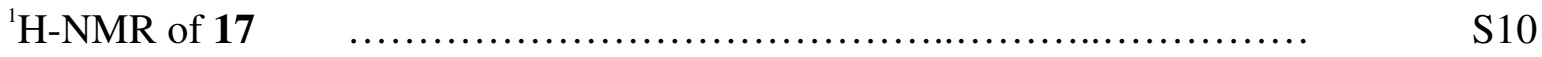

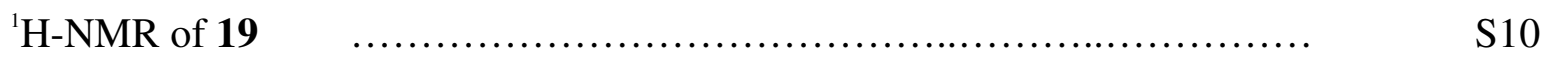

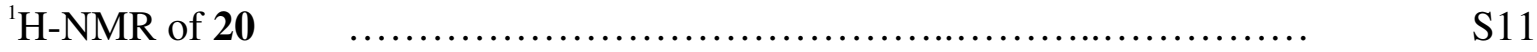

${ }^{1} \mathrm{H}-\mathrm{NMR}$ of 21 


\section{General Methods.}

Anhydrous solvents and reagents were reagent grade items used without prior purification, but with the following exception: 1,2-dichloroethane was used as is, or with purification (shaken with $\mathrm{H}_{2} \mathrm{SO}_{4}$, water, $1 \mathrm{M} \mathrm{KOH}$, dried over $\mathrm{MgSO}_{4}$, then distilled from $\mathrm{CaH}$ ). All reactions were performed under either nitrogen or argon atmosphere unless otherwise indicated.

The EM Science precoated thin layer chromatography (TLC) plates (silica gel $60 \mathrm{~F}_{254}$ ) were used for normal phase TLC: $0.25 \mathrm{~mm}$ thickness for analytical TLC analyses, $2 \mathrm{~mm}$ thickness for preparative TLC (PTLC) purification. Visualization of compounds was done with a UV lamp. Silica gel used for column chromatography was the EM Science silica gel 60 (70 - 230 mesh).

High performance liquid chromatography (HPLC) was performed using a Zorbax SB-C8 column $(25 \mathrm{~cm} \times 4.6 \mathrm{~mm})$ with UV detection at $235 \mathrm{~nm}$. Two solvents were required: Solvent A containing 80:20:0.1 water/acetonitrile/85\% $\quad \mathrm{H}_{3} \mathrm{PO}_{4} \quad$ and Solvent $\mathrm{B}$ containing 80:20:0.1 acetonitrile/water/85\% $\mathrm{H}_{3} \mathrm{PO}_{4}$. The linear gradient changed from $100 \%$ solvent $\mathrm{A}$ to $100 \%$ solvent $\mathrm{B}$ over the course of the $30 \mathrm{~min}$ cycle. The flow rate was $1.0 \mathrm{~mL} / \mathrm{min}$.

All ${ }^{1} \mathrm{H}-\mathrm{NMR}$ spectra were obtained at $400 \mathrm{MHz}$, and ${ }^{13} \mathrm{C}-\mathrm{NMR}$ at $100 \mathrm{MHz}$. For ${ }^{1} \mathrm{H}-\mathrm{NMR}$, multiplicity ( $\mathrm{s}=$ singlet, $\mathrm{d}=$ doublet, $\mathrm{t}=$ triplet, $\mathrm{m}=$ multiplet $)$ and coupling constant(s) (in Hz) were reported whenever possible. ${ }^{13} \mathrm{C}$-NMR spectra were recorded with complete proton decoupling. 
3-Vinylquinoline (6). To a suspension of methyltriphenylphosphonium bromide (56 mmol) in THF (112 mL) at $<4{ }^{\circ} \mathrm{C}$ was slowly added potassium bis(trimethylsilyl)amide (56 mmol, $0.5 \mathrm{M}$ in toluene) while maintaining the internal temperature at $<5{ }^{\circ} \mathrm{C}$. Quinoline-3-carboxaldehyde $(50 \mathrm{mmol})$ was then added in portions. The progress was monitored by TLC (3:2 Hept/EtOAc). When the reaction was complete, it was quenched with saturated $\mathrm{NH}_{4} \mathrm{Cl}(100 \mathrm{~mL})$ and water $(100 \mathrm{~mL})$. The organic layer was washed with saturated $\mathrm{NaCl}(100 \mathrm{~mL})$ and concentrated. The desired product was isolated by silica gel chromatography, eluting with 2:1 Hept/EtOAc. Yield $=98 \% .{ }^{1} \mathrm{H}-\mathrm{NMR}\left(\mathrm{CDCl}_{3}, 400 \mathrm{MHz}\right): 9.02$ $\operatorname{ppm}(\mathrm{d}, J=2.2 \mathrm{~Hz}, 1 \mathrm{H}), 8.09(\mathrm{~d}, J=8.4 \mathrm{~Hz}, 1 \mathrm{H}), 8.06(\mathrm{~d}, J=2.2 \mathrm{~Hz}, 1 \mathrm{H}), 7.79(\mathrm{dd}, J=1.0,8.1,1 \mathrm{H})$, $7.68(\mathrm{~m}, 1 \mathrm{H}), 7.53(\mathrm{~m}, 1 \mathrm{H}), 6.87(\mathrm{dd}, J=11.0,17.7 \mathrm{~Hz}, 1 \mathrm{H}), 5.98(\mathrm{~d}, J=17.7 \mathrm{~Hz}, 1 \mathrm{H}), 5.46(\mathrm{~d}, J=$ 11.1 Hz, 1H). ${ }^{13} \mathrm{C}-\mathrm{NMR}(\mathrm{CDCl} 3,100 \mathrm{MHz}): 148.4 \mathrm{ppm}, 146.9,133.2,132.3,130.0,129.0,128.6$, $127.6,127.5,126.6,116.1$.

Compound 10. A solution of $\mathbf{8}(377 \mathrm{mg})$, styrene $(0.11 \mathrm{~mL})$, and $\mathbf{3}$ (43 mg) in methylene chloride $(5.0 \mathrm{~mL})$ was brought to a reflux under nitrogen atmosphere for 26 hours. The solvent was removed under reduced pressure, and $\mathbf{1 0}$ was isolated by silica gel chromatography (EtOAc). Yield $=81 \%$. ${ }^{1} \mathrm{H}-$ $\operatorname{NMR}\left(\mathrm{CDCl}_{3}, 400 \mathrm{MHz}\right): 8.05 \mathrm{ppm}(\mathrm{m}, 2 \mathrm{H}), 7.55(\mathrm{~m}, 1 \mathrm{H}), 7.52-7.41(\mathrm{~m}, 4 \mathrm{H}), 7.30(\mathrm{~m}, 2 \mathrm{H}), 7.27-$ $7.18(\mathrm{~m}, 1 \mathrm{H}), 6.48(\mathrm{~d}, J=15.8 \mathrm{~Hz}, 1 \mathrm{H}), 6.09(\mathrm{~m}, 1 \mathrm{H}), 5.25(\mathrm{~s}, 1 \mathrm{H}), 5.06(\mathrm{~m}, 2 \mathrm{H}), 4.81(\mathrm{~d}, J=7.5 \mathrm{~Hz}$, 1H), $3.92-3.65(\mathrm{~m}, 4 \mathrm{H}), 3.60-3.47(\mathrm{~m}, 2 \mathrm{H}), 2.90(\mathrm{~m}, 1 \mathrm{H}), 2.79(\mathrm{~m}, 1 \mathrm{H}), 2.67-2.46(\mathrm{~m}, 2 \mathrm{H}), 2.28$ (s, 6H), $2.05-1.61(\mathrm{~m}, 4 \mathrm{H}), 1.15-1.06(\mathrm{~m}, 3 \mathrm{H}), 1.37(\mathrm{~s}, 3 \mathrm{H}), 1.30(\mathrm{~s}, 3 \mathrm{H}), 1.27(\mathrm{~s}, 3 \mathrm{H}), 1.25(\mathrm{~s}, 3 \mathrm{H})$, $1.03(\mathrm{~d}, J=7.1 \mathrm{~Hz}, 3 \mathrm{H}), 1.03(\mathrm{~d}, J=6.6,3 \mathrm{H}), 0.83(\mathrm{t}, J=7.4 \mathrm{~Hz}, 3 \mathrm{H}), 0.74(\mathrm{~d}, J=7.4 \mathrm{~Hz}, 3 \mathrm{H}) .{ }^{13} \mathrm{C}-$ $\mathrm{NMR}\left(\mathrm{CDCl}_{3}, 100 \mathrm{MHz}\right): 216.3 \mathrm{ppm}, 174.4,164.9,157.3,136.2,133.2,132.4,130.3,129.4,128.3$, $128.0,127.5,126.2,125.0,99.4,83.6,81.0,78.6,77.8,75.8,72.2,69.0,64.2,63.3,58.2,45.7,44.0$, 41.0, 38.5, 37.4, 35.9, 32.2, 22.7, 21.4, 20.4, 18.7, 15.5, 14.2, 13.7, 10.9, 8.1. HR-MS (ESI + MS) calculated for $\left(\mathrm{C}_{46} \mathrm{H}_{65} \mathrm{~N}_{2} \mathrm{O}_{11}\right)^{+}: 821.4583$, found 821.4596 .

3-Vinylquinoline Dimer (11). To a solution of 6 (155 mg) in methylene chloride (10.0 mL) was added Grubbs' second generation catalyst $^{8}(23 \mathrm{mg})$. After refluxing for 3 days under nitrogen atmosphere, more catalyst $(51 \mathrm{mg})$ and solvent $(5.0 \mathrm{~mL})$ were added. The mixture was heated at reflux 
for another day before all the starting material was consumed. The solvent was removed under reduced pressure, and the product was isolated by silica gel chromatography (EtOAc). ${ }^{1} \mathrm{H}-\mathrm{NMR}$ $\left(\mathrm{CDCl}_{3}, 400 \mathrm{MHz}\right): 9.12 \mathrm{ppm}(\mathrm{d}, J=2.2 \mathrm{~Hz}, 2 \mathrm{H}), 8.19(\mathrm{~d}, J=2.0 \mathrm{~Hz}, 2 \mathrm{H}), 8.05(\mathrm{~d}, J=8.3 \mathrm{~Hz}, 2 \mathrm{H})$, $7.80(\mathrm{dd}, J=1.0,8.1 \mathrm{~Hz}, 2 \mathrm{H}), 7.65(\mathrm{~m}, 2 \mathrm{H}), 7.51(\mathrm{~m}, 2 \mathrm{H}), 7.41(\mathrm{~s}, 2 \mathrm{H}) .{ }^{13} \mathrm{C}-\mathrm{NMR}\left(\mathrm{CDCl}_{3}, 100 \mathrm{MHz}\right)$ : 149.4 ppm, 147.9, 133.0, 129.9, 129.7, 129.5, 128.2, 128.1, 127.6, 127.3.

Compound 16. To a solution of $8(377 \mathrm{mg})$ and 1-pentene $(0.55 \mathrm{~mL})$ in methylene chloride $(5.0$ $\mathrm{mL}$ ) was added 3 (44 mg). After refluxing for $\sim 20 \mathrm{hrs,} \mathrm{the} \mathrm{solvent} \mathrm{was} \mathrm{removed} \mathrm{under} \mathrm{reduced}$ pressure, and the product was isolated by silica gel chromatography (EtOAc). Yield $=84 \%$. ${ }^{1} \mathrm{H}-\mathrm{NMR}$ $\left(\mathrm{CDCl}_{3}, 400 \mathrm{MHz}\right): 8.05 \mathrm{ppm}(\mathrm{d}, J=8.0 \mathrm{~Hz}, 2 \mathrm{H}), 7.56(\mathrm{t}, J=7.4,1 \mathrm{H}), 7.44(\mathrm{t}, J=7.6,2 \mathrm{H}), 5.58(\mathrm{~m}$, $1 \mathrm{H}), 5.47(\mathrm{~m}, 1 \mathrm{H}), 5.28(\mathrm{~m}, 1 \mathrm{H}), 5.13(\mathrm{ddd}, J=2.7,10.3,18.7,1 \mathrm{H}), 5.03(\mathrm{ddd}, J=2.7,7.7,10.3,1 \mathrm{H})$, $4.79(\mathrm{~d}, J=7.6,1 \mathrm{H}), 3.77-3.40(\mathrm{~m}, 5 \mathrm{H}), 2.89(\mathrm{~m}, 1 \mathrm{H}), 2.80(\mathrm{~m}, 1 \mathrm{H}), 2.60(\mathrm{~m}, 1 \mathrm{H}), 2.49(\mathrm{~m}, 1 \mathrm{H})$, $2.27(\mathrm{~s}, 6 \mathrm{H}), 2.07-1.58(\mathrm{~m}, 7 \mathrm{H}), 1.52-1.18(\mathrm{~m}, 5 \mathrm{H}), 1.33(\mathrm{~s}, 3 \mathrm{H}), 1.32(\mathrm{~d}, J=6.2,3 \mathrm{H}), 1.26(\mathrm{~d}, J=$ $6.3,3 \mathrm{H}), 1.24(\mathrm{~d}, J=7.6,3 \mathrm{H}), 1.08(\mathrm{~d}, J=6.6,3 \mathrm{H}), 1.02(\mathrm{~d}, J=7.1,3 \mathrm{H}), 0.91(\mathrm{t}, J=7.4,3 \mathrm{H}), 0.83(\mathrm{t}$, $J=7.3,3 \mathrm{H}), 0.72(\mathrm{~d}, J=7.4,3 \mathrm{H}) .{ }^{13} \mathrm{C}-\mathrm{NMR}\left(\mathrm{CDCl}_{3}, 100 \mathrm{MHz}\right): 216.3 \mathrm{ppm}, 174.1,164.9,157.8$, $134.8,132.4,132.2,130.4,129.4,128.0,125.5,99.5,83.8,81.0,78.3,77.8,75.5,72.2,69.0,64.2$, $63.3,58.0,45.6,43.9,41.0,38.5,37.4,35.8,34.6,32.2,22.8,22.5,21.4,20.4,18.6,15.5,14.2,14.1$, 13.7, 10.8, 10.7, 8.1. HR-MS (ESI + MS) calculated for $\left(\mathrm{C}_{43} \mathrm{H}_{67} \mathrm{~N}_{2} \mathrm{O}_{11}\right)^{+}:$787.4740, found 787.4746.

Compound 17. To a solution of $6(78 \mathrm{mg})$ and 1-pentene $(0.55 \mathrm{~mL})$ in methylene chloride $(5.0 \mathrm{~mL})$ was added Grubbs' second generation catalyst ${ }^{8}(43 \mathrm{mg})$. After refluxing for $\sim 20 \mathrm{hrs}$, the solvent was removed under reduced pressure, and the product was isolated by preparative TLC (9:1 Hept/EtOAc). Yield $=73 \% .{ }^{1} \mathrm{H}-\mathrm{NMR}\left(\mathrm{CDCl}_{3}, 400 \mathrm{MHz}\right): 8.95 \mathrm{ppm}(\mathrm{d}, J=2.2 \mathrm{~Hz}, 1 \mathrm{H}), 8.04(\mathrm{~d}, J=8.4,1 \mathrm{H}), 7.96$ $(\mathrm{d}, J=1.9,1 \mathrm{H}), 7.75(\mathrm{dd}, J=1.4,8.1,1 \mathrm{H}), 7.62(\mathrm{~m}, 1 \mathrm{H}), 7.49(\mathrm{~m}, 1 \mathrm{H}), 6.55-6.40(\mathrm{~m}, 2 \mathrm{H}), 2.27(\mathrm{dt}$, $J=6.4,6.5,2 \mathrm{H}), 1.55(\mathrm{~m}, 2 \mathrm{H}), 0.99(\mathrm{t}, J=7.4,3 \mathrm{H}) .{ }^{13} \mathrm{C}-\mathrm{NMR}\left(\mathrm{CDCl}_{3}, 100 \mathrm{MHz}\right): 149.0 \mathrm{ppm}, 146.8$, $133.4,131.1,130.4,128.9,128.4,127.8,127.3,126.5,125.4,35.6,22.7,14.1$.

Compound 19. A solution of $18(370 \mathrm{mg})$, styrene $(0.11 \mathrm{~mL})$, and $\mathbf{3}(44 \mathrm{mg})$ in methylene chloride $(5.0 \mathrm{~mL})$ was heated to reflux under nitrogen atmosphere. An additional portion of $\mathbf{3}$ (40 $\mathrm{mg}$ each) 
was added after one day. To push the reaction to completion, more styrene $(0.50 \mathrm{~mL})$ and 3 (43 $\mathrm{mg})$ were added after one more day. Heating was continued for six more hours after those reagents were charged. After concentrating the reaction mixture, the product was isolated by silica gel chromatography (EtOAc). The purity was further enhanced by preparative-TLC (EtOAc). Yield = 52\%. ${ }^{1} \mathrm{H}-\mathrm{NMR}\left(\mathrm{CDCl}_{3}, 400 \mathrm{MHz}\right): 8.04-7.97 \mathrm{ppm}(\mathrm{m}, 2 \mathrm{H}), 7.57-7.51(\mathrm{~m}, 1 \mathrm{H}), 7.45-7.38(\mathrm{~m}$, 4H), $7.34-7.27(\mathrm{~m}, 2 \mathrm{H}), 7.24-7.18(\mathrm{~m}, 1 \mathrm{H}), 6.39(\mathrm{~d}, J=15.8 \mathrm{~Hz}, 1 \mathrm{H}), 5.91(\mathrm{dt}, J=6.9,15.6 \mathrm{~Hz}$ 1H), $5.29(\mathrm{~s}, 1 \mathrm{H}), 5.03(\mathrm{dd}, J=7.7,10.4 \mathrm{~Hz}, 1 \mathrm{H}), 4.85(\mathrm{dd}, J=3.5,8.8 \mathrm{~Hz}, 1 \mathrm{H}), 4.58(\mathrm{~d}, J=7.7 \mathrm{~Hz}$, $1 \mathrm{H}), 4.36(\mathrm{~d}, J=4.3,1 \mathrm{H}), 3.87-3.72(\mathrm{~m}, 3 \mathrm{H}), 3.72-3.52(\mathrm{~m}, 2 \mathrm{H}), 3.07(\mathrm{~m}, 1 \mathrm{H}), 2.93-2.78(\mathrm{~m}$, 2H), $2.65-2.52(\mathrm{~m}, 1 \mathrm{H}), 2.27(\mathrm{~s}, 6 \mathrm{H}), 1.92-1.75(\mathrm{~m}, 2 \mathrm{H}), 1.64-1.20(\mathrm{~m}, 4 \mathrm{H}), 1.41(\mathrm{~s}, 3 \mathrm{H}), 1.38(\mathrm{~s}$, $3 \mathrm{H}), 1.35(\mathrm{~d}, J=6.7 \mathrm{~Hz}, 3 \mathrm{H}), 1.24(\mathrm{~d}, J=6.0 \mathrm{~Hz}, 3 \mathrm{H}), 1.10(\mathrm{~d}, J=7.1 \mathrm{~Hz}, 3 \mathrm{H}), 1.06(\mathrm{~d}, J=6.6 \mathrm{~Hz}$, 3H), $1.00(\mathrm{~d}, J=7.7 \mathrm{~Hz}, 3 \mathrm{H}), 0.81(\mathrm{t}, J=7.5 \mathrm{~Hz}, 3 \mathrm{H}) .{ }^{13} \mathrm{C}-\mathrm{NMR}\left(\mathrm{CDCl}_{3}, 100 \mathrm{MHz}\right): 216.4 \mathrm{ppm}$, $204.7,168.9,164.7,156.9,136.1,133.4,132.5,130.2,129.4,128.3,128.0,127.5,126.2,125.1,100.6$, 83.3, 78.4, 77.7, 75.6, 72.0, 69.2, 64.3, 63.5, 58.3, 50.9, 45.9, 45.2, 40.9, 38.9, 37.5, 31.8, 23.0, 21.3, 20.5, 18.4, 14.7, 14.2, 14.0, 14.0, 11.1. HR-MS (ESI + MS) calculated for $\left(\mathrm{C}_{46} \mathrm{H}_{63} \mathrm{~N}_{2} \mathrm{O}_{11}\right)^{+}:$819.4406, found 819.4406 .

Compound 20. A solution of $8(372 \mathrm{mg}), 3$-chlorostyrene $(0.13 \mathrm{~mL})$, and $\mathbf{3}(41 \mathrm{mg})$ in methylene chloride $(25 \mathrm{~mL})$ was heated to reflux under nitrogen atmosphere. After 65 hours the reaction was incomplete, so more 3 (41 mg) was added. The mixture was refluxed for another 20 hours before it was concentrated. The product was isolated by silica gel chromatography (95:5 methylene chloride / methanol). Yield $=81.3 \% .{ }^{1} \mathrm{H}-\mathrm{NMR}\left(\mathrm{CDCl}_{3}, 400 \mathrm{MHz}\right): 8.02-7.96 \mathrm{ppm}(\mathrm{m}, 2 \mathrm{H}), 7.50(\mathrm{~m}, 1 \mathrm{H})$, $7.44-7.35(\mathrm{~m}, 3 \mathrm{H}), 7.25(\mathrm{~m}, 1 \mathrm{H}), 7.20-7.09(\mathrm{~m}, 2 \mathrm{H}), 6.35(\mathrm{~d}, J=15.9 \mathrm{~Hz}, 1 \mathrm{H}), 6.05(\mathrm{dt}, J=6.2$, $15.8 \mathrm{~Hz}, 1 \mathrm{H}), 5.19(\mathrm{~s}, 1 \mathrm{H}), 5.05-4.95(\mathrm{~m}, 2 \mathrm{H}), 4.75(\mathrm{~d}, J=7.3 \mathrm{~Hz}, 1 \mathrm{H}), 3.83-3.55(\mathrm{~m}, 4 \mathrm{H}), 3.55-$ $3.38(\mathrm{~m}, 2 \mathrm{H}), 2.99-2.78(\mathrm{~m}, 1 \mathrm{H}), 2.72(\mathrm{~m}, 1 \mathrm{H}), 2.62-2.39(\mathrm{~m}, 2 \mathrm{H}), 2.27(\mathrm{~s}, 6 \mathrm{H}), 1.95-1.53(\mathrm{~m}$, 4H), $1.44-0.95(\mathrm{~m}, 3 \mathrm{H}), 1.30(\mathrm{~s}, 3 \mathrm{H}), 1.23(\mathrm{~s}, 3 \mathrm{H}), 1.20(\mathrm{~d}, J=6.3 \mathrm{~Hz}, 6 \mathrm{H}), 0.97(\mathrm{~d}, J=7.3 \mathrm{~Hz}, 3 \mathrm{H})$, $0.96(\mathrm{~d}, J=6.4 \mathrm{~Hz}, 3 \mathrm{H}), 0.77(\mathrm{t}, J=7.4 \mathrm{~Hz}, 3 \mathrm{H}), 0.66(\mathrm{~d}, J=7.3 \mathrm{~Hz}, 3 \mathrm{H}) .{ }^{13} \mathrm{C}-\mathrm{NMR}\left(\mathrm{CDCl}_{3}, 100\right.$ MHz): 174.4 ppm, 157.3, 134.2, 131.7, 129.6, 129.5, 128.1, 127.5, 126.7, 126.2, 124.4, 83.5, 80.9, 
78.7, 77.9, 75.9, 64.0, 63.4, 58.2, 45.7, 44.0, 38.5, 37.4, 35.9, 27.3, 27.1, 22.7, 21.4, 20.4, 18.7, 15.5, 14.2, 13.7, 10.8, 8.1. HR-MS (ESI + MS) calculated for $\left(\mathrm{C}_{46} \mathrm{H}_{64} \mathrm{ClN}_{2} \mathrm{O}_{11}\right)^{+}:$855.4193, found 855.4183.

Compound 21. A solution of $8(372 \mathrm{mg})$, 4-methylstyrene $(0.13 \mathrm{~mL})$, and $\mathbf{3}(41 \mathrm{mg})$ in methylene chloride $(25 \mathrm{~mL})$ was heated to reflux under nitrogen atmosphere. After 65 hours the reaction was incomplete, so more 3 (41 mg) was added. The mixture was refluxed for another $\sim 20$ hours before it was concentrated. The product was isolated by silica gel chromatography (95:5 methylene chloride / methanol). $\quad$ Yield $=91.5 \% . \quad{ }^{1} \mathrm{H}-\mathrm{NMR}\left(\mathrm{CDCl}_{3}, 400 \mathrm{MHz}\right): 8.11-8.05 \mathrm{ppm}(\mathrm{m}, 2 \mathrm{H}), 7.60(\mathrm{~m}, 1 \mathrm{H})$, $7.51-7.44(\mathrm{~m}, 2 \mathrm{H}), 7.40-7.34(\mathrm{~m}, 2 \mathrm{H}), 7.16-7.11(\mathrm{~m}, 2 \mathrm{H}), 6.47(\mathrm{~d}, J=15.8 \mathrm{~Hz}, 1 \mathrm{H}), 6.05(\mathrm{dt}, J=$ $6.9,15.9,1 \mathrm{H}), 5.31(\mathrm{~s}, 1 \mathrm{H}), 5.28(\mathrm{~s}, 1 \mathrm{H}), 5.11(\mathrm{dd}, J=2.8,10.2,1 \mathrm{H}), 4.84(\mathrm{~d}, J=7.8,1 \mathrm{H}), 3.92-3.76$ (m, 3H), $3.73(\mathrm{~s}, 1 \mathrm{H}), 3.64-3.49(\mathrm{~m}, 2 \mathrm{H}), 2.80(\mathrm{~m}, 1 \mathrm{H}), 2.70-2.48(\mathrm{~m}, 2 \mathrm{H}), 2.41-2.30(\mathrm{~m}, 4 \mathrm{H})$, $2.33(\mathrm{~s}, 6 \mathrm{H}), 2.06-1.62(\mathrm{~m}, 4 \mathrm{H}), 1.53-1.21(\mathrm{~m}, 3 \mathrm{H}), 1.39(\mathrm{~s}, 3 \mathrm{H}), 1.32(\mathrm{~s}, 3 \mathrm{H}), 1.29(\mathrm{~d}, J=5.1,3 \mathrm{H})$, $1.29(\mathrm{~d}, J=6.7,3 \mathrm{H}), 1.05(\mathrm{~d}, J=6.5,3 \mathrm{H}), 1.05(\mathrm{~d}, J=8.2,3 \mathrm{H}), 0.85(\mathrm{t}, J=7.3,3 \mathrm{H}), 0.74(\mathrm{~d}, J=7.3$, 3H). ${ }^{13} \mathrm{C}-\mathrm{NMR}\left(\mathrm{CDCl}_{3}, 100 \mathrm{MHz}\right): 174.3 \mathrm{ppm}, 157.3,137.2,133.5,133.3,129.5,129.1,128.1,126.1$, $123.8,83.6,81.0,78.5,75.8,64.3,58.2,45.7,44.0,37.4,26.6,22.8,21.5,20.5,18.7,15.5,14.2,13.7$, 10.9, 8.1. HR-MS (ESI + MS) calculated for $\left(\mathrm{C}_{47} \mathrm{H}_{67} \mathrm{~N}_{2} \mathrm{O}_{11}\right)^{+}: 835.4739$, found 835.4743. 
${ }^{1} \mathrm{H}-\mathrm{NMR}$ of Compound $\mathbf{6}\left(\mathrm{CDCl}_{3}, 400 \mathrm{MHz}\right)$

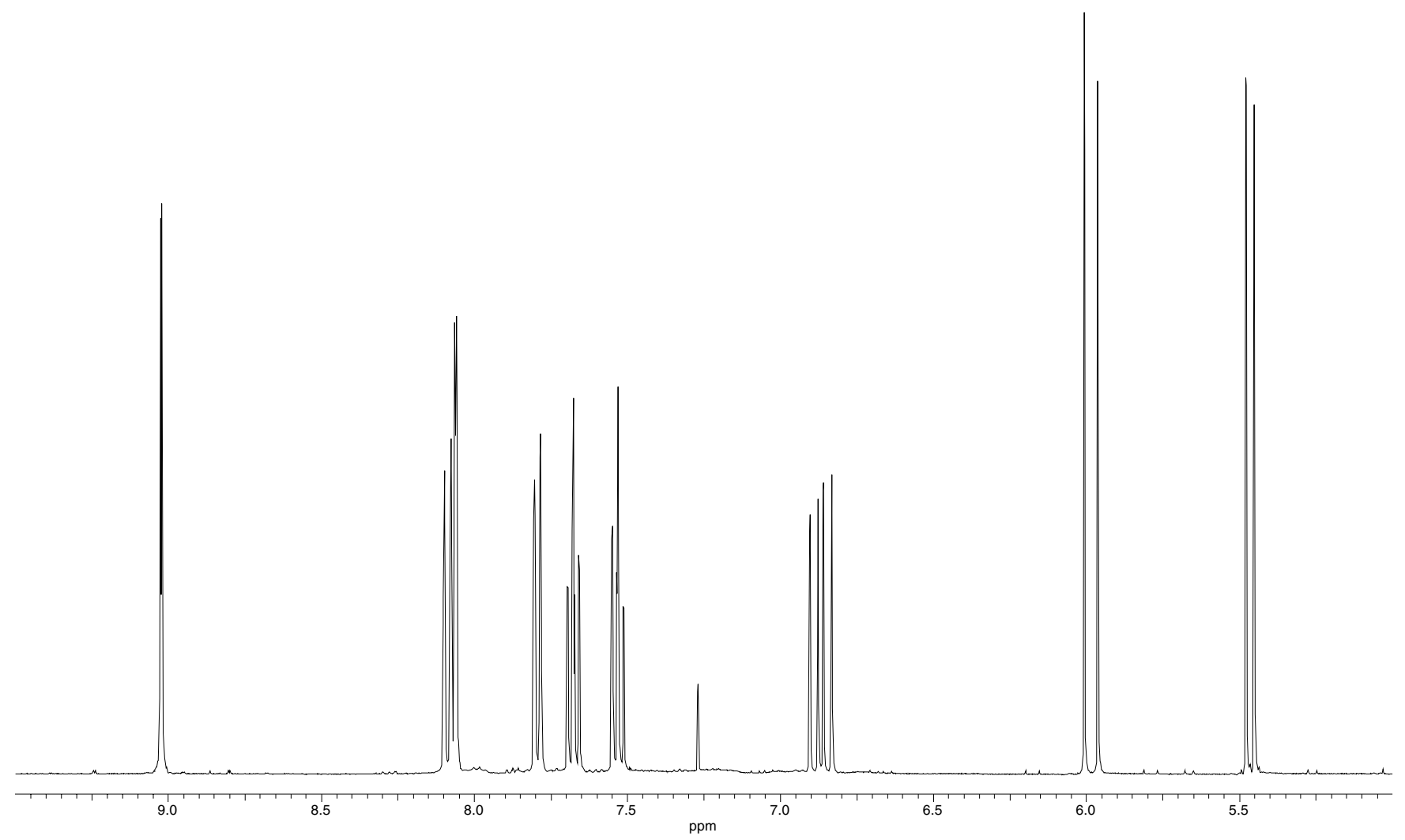

${ }^{1} \mathrm{H}-\mathrm{NMR}$ of Compound $\mathbf{1 0}\left(\mathrm{CDCl}_{3}, 400 \mathrm{MHz}\right)$

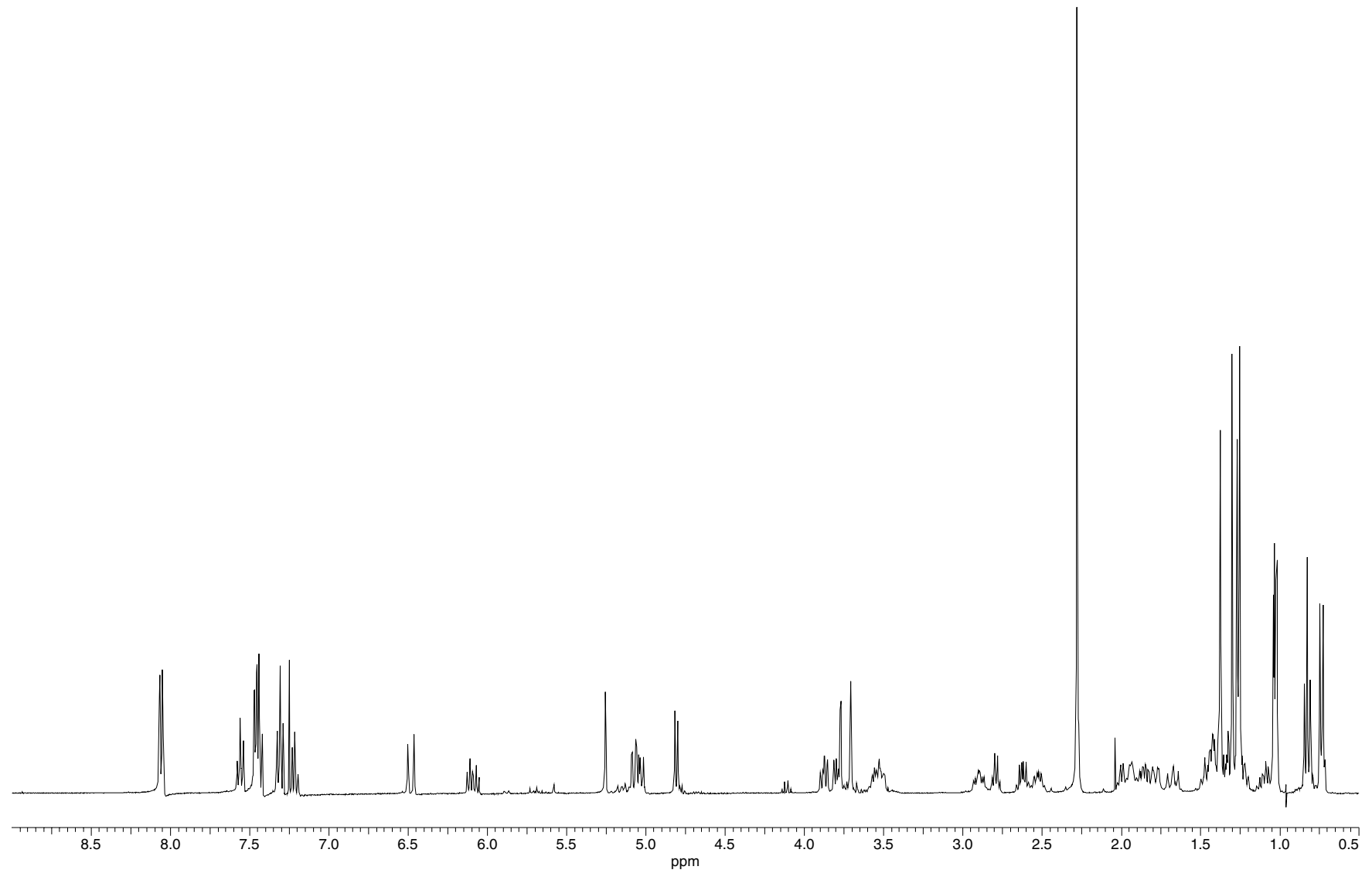


${ }^{1} \mathrm{H}-\mathrm{NMR}$ of Compound $11\left(\mathrm{CDCl}_{3}, 400 \mathrm{MHz}\right)$

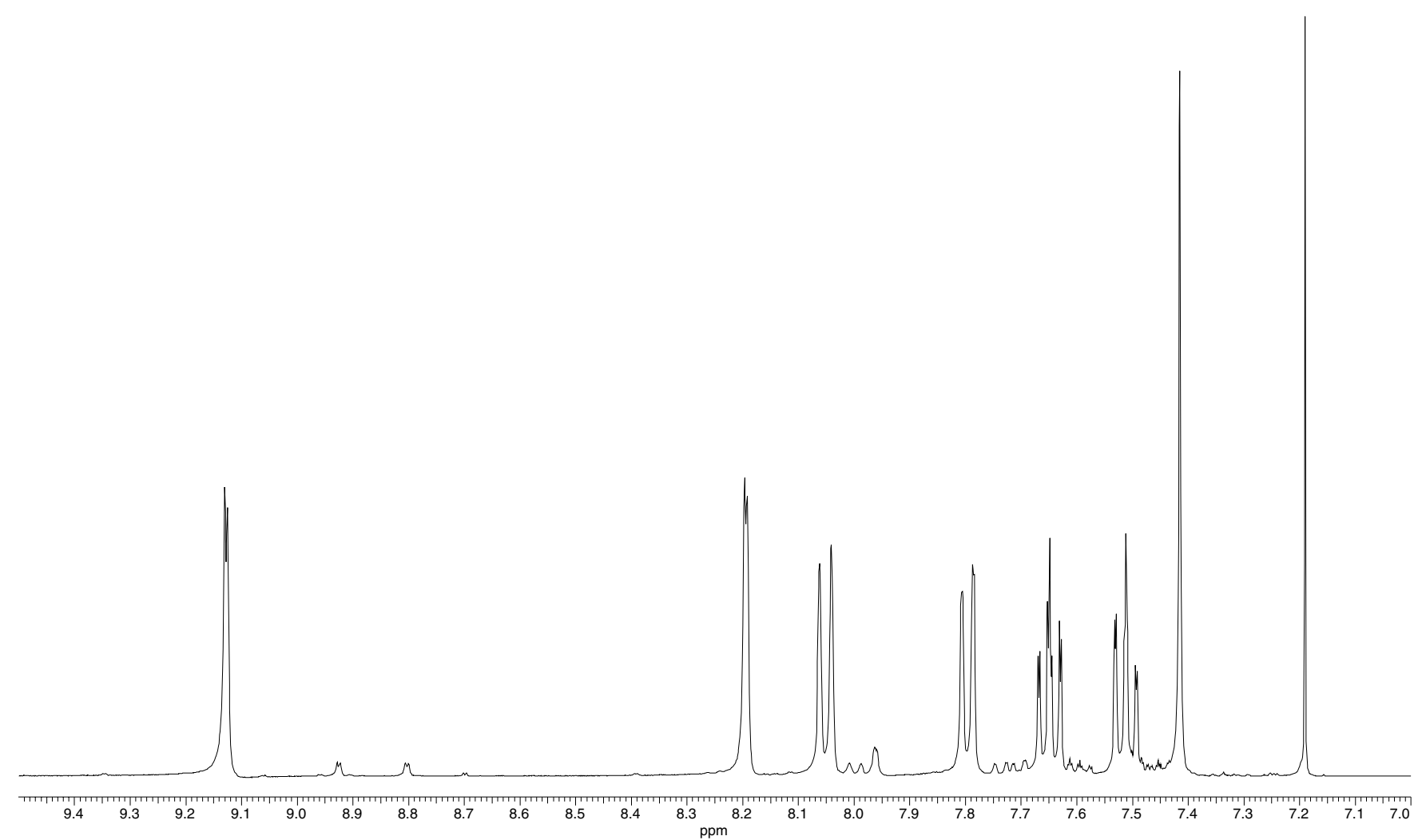

${ }^{1} \mathrm{H}-\mathrm{NMR}$ of Compound $16\left(\mathrm{CDCl}_{3}, 400 \mathrm{MHz}\right)$

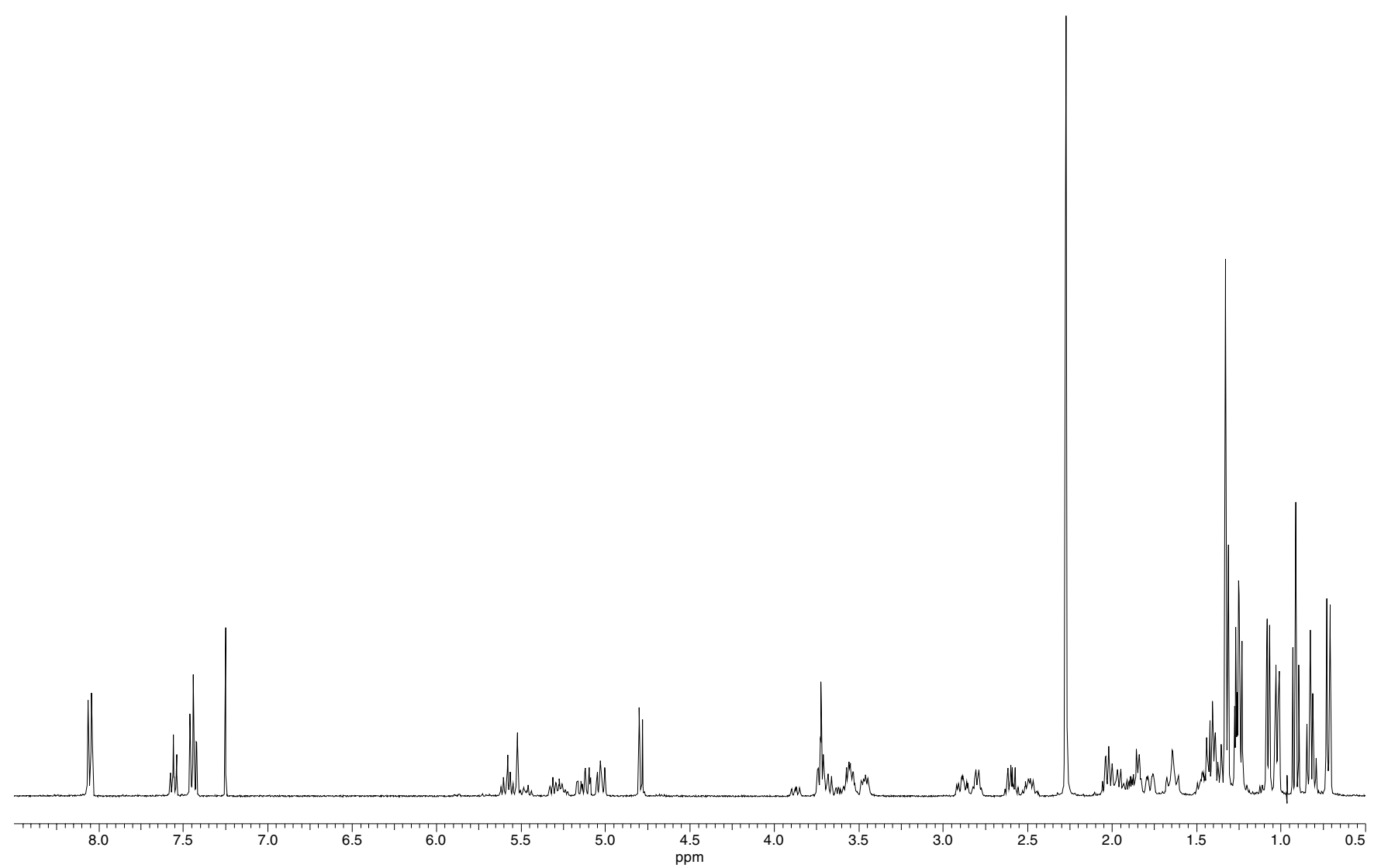


${ }^{1} \mathrm{H}-\mathrm{NMR}$ of Compound $17\left(\mathrm{CDCl}_{3}, 400 \mathrm{MHz}\right)$

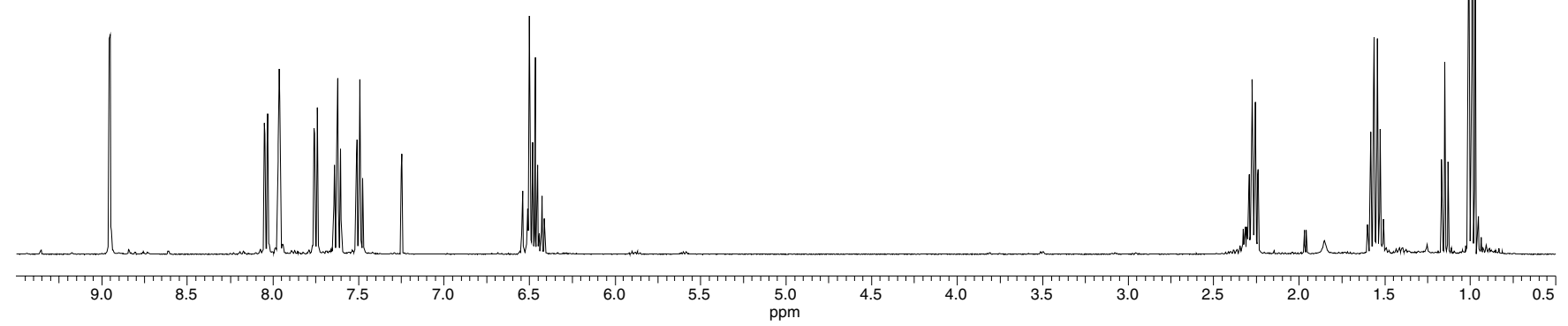

${ }^{1} \mathrm{H}-\mathrm{NMR}$ of Compound $19\left(\mathrm{CDCl}_{3}, 400 \mathrm{MHz}\right)$

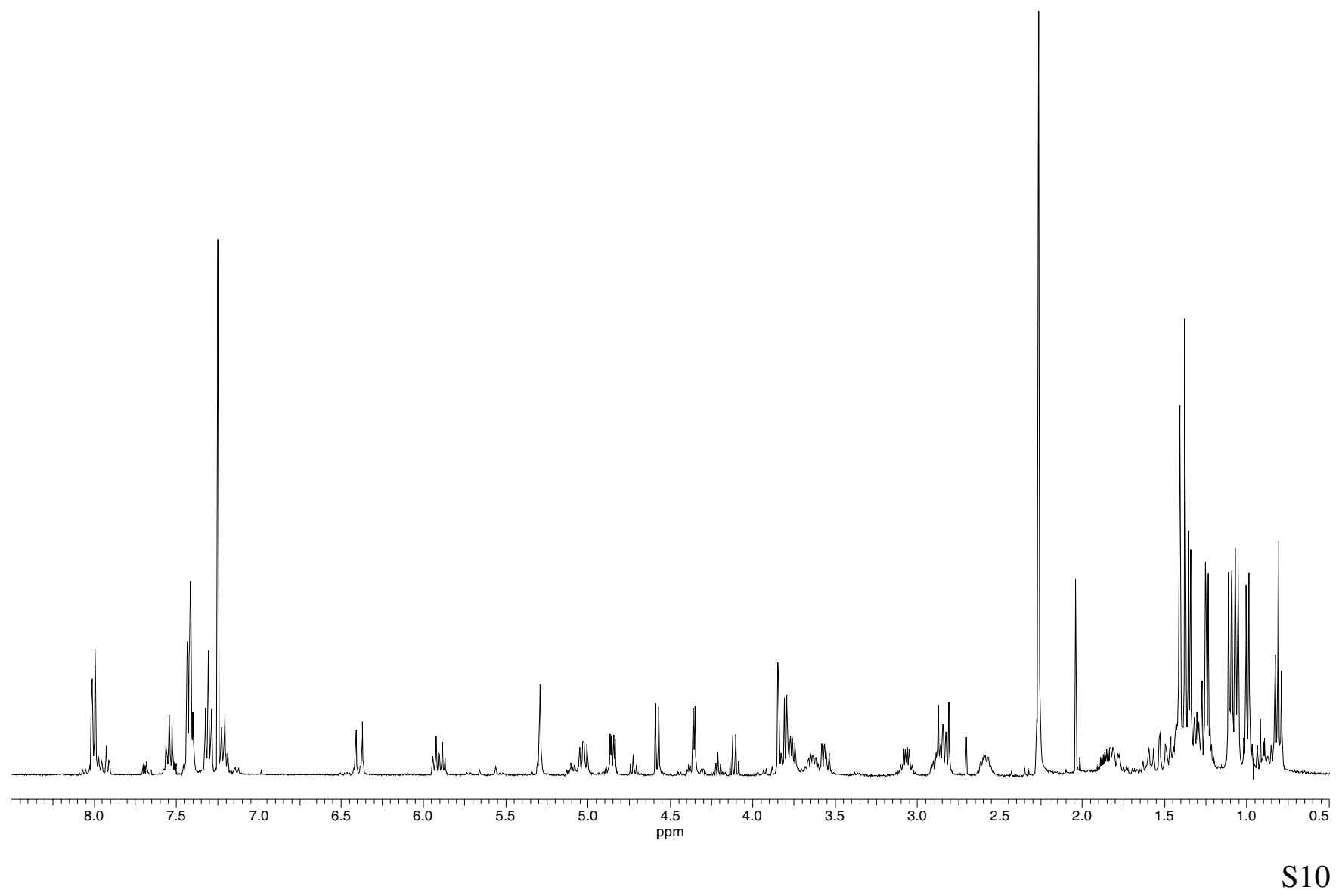


${ }^{1} \mathrm{H}-\mathrm{NMR}$ of Compound $20\left(\mathrm{CDCl}_{3}, 400 \mathrm{MHz}\right)$

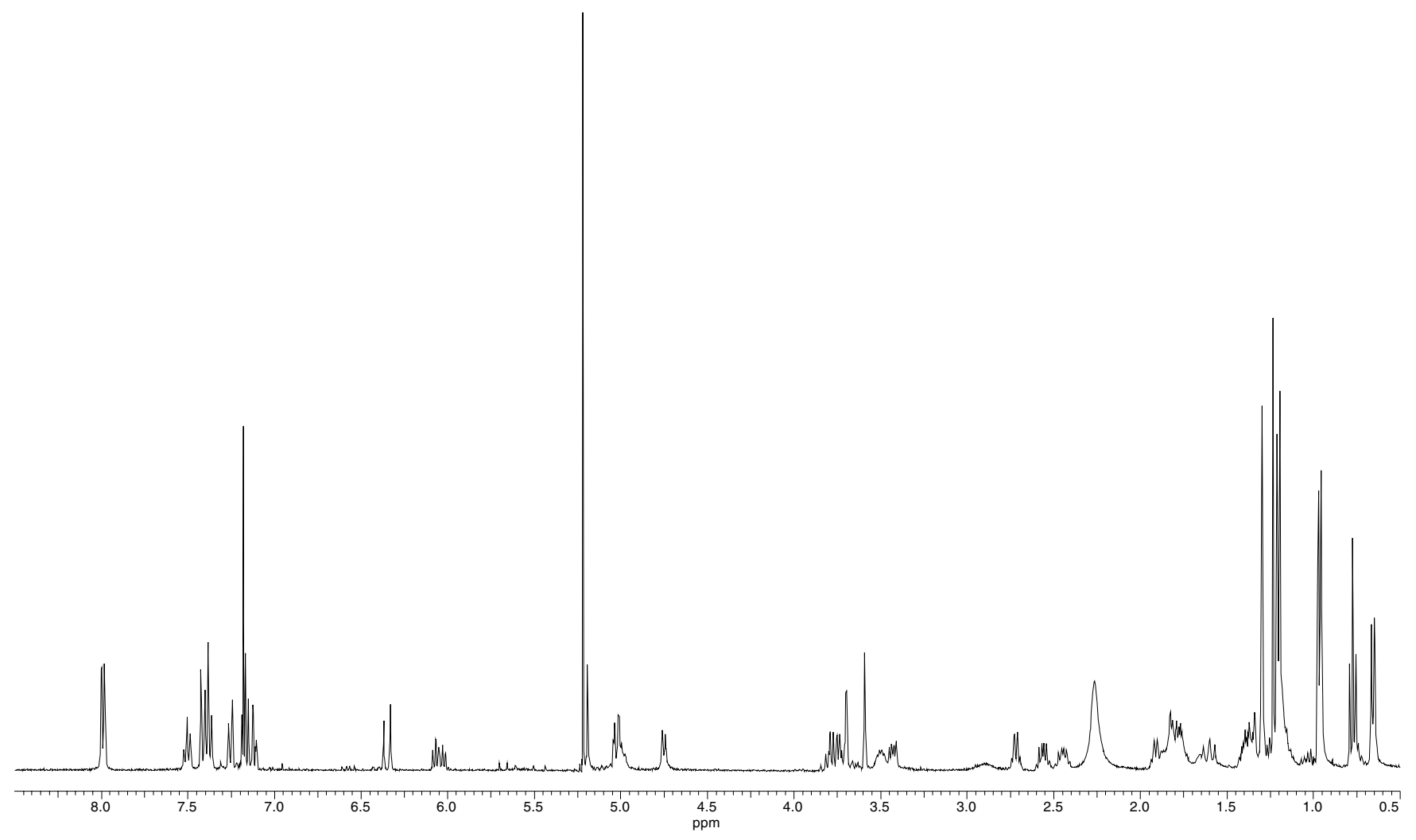

${ }^{1} \mathrm{H}-\mathrm{NMR}$ of Compound $21\left(\mathrm{CDCl}_{3}, 400 \mathrm{MHz}\right)$

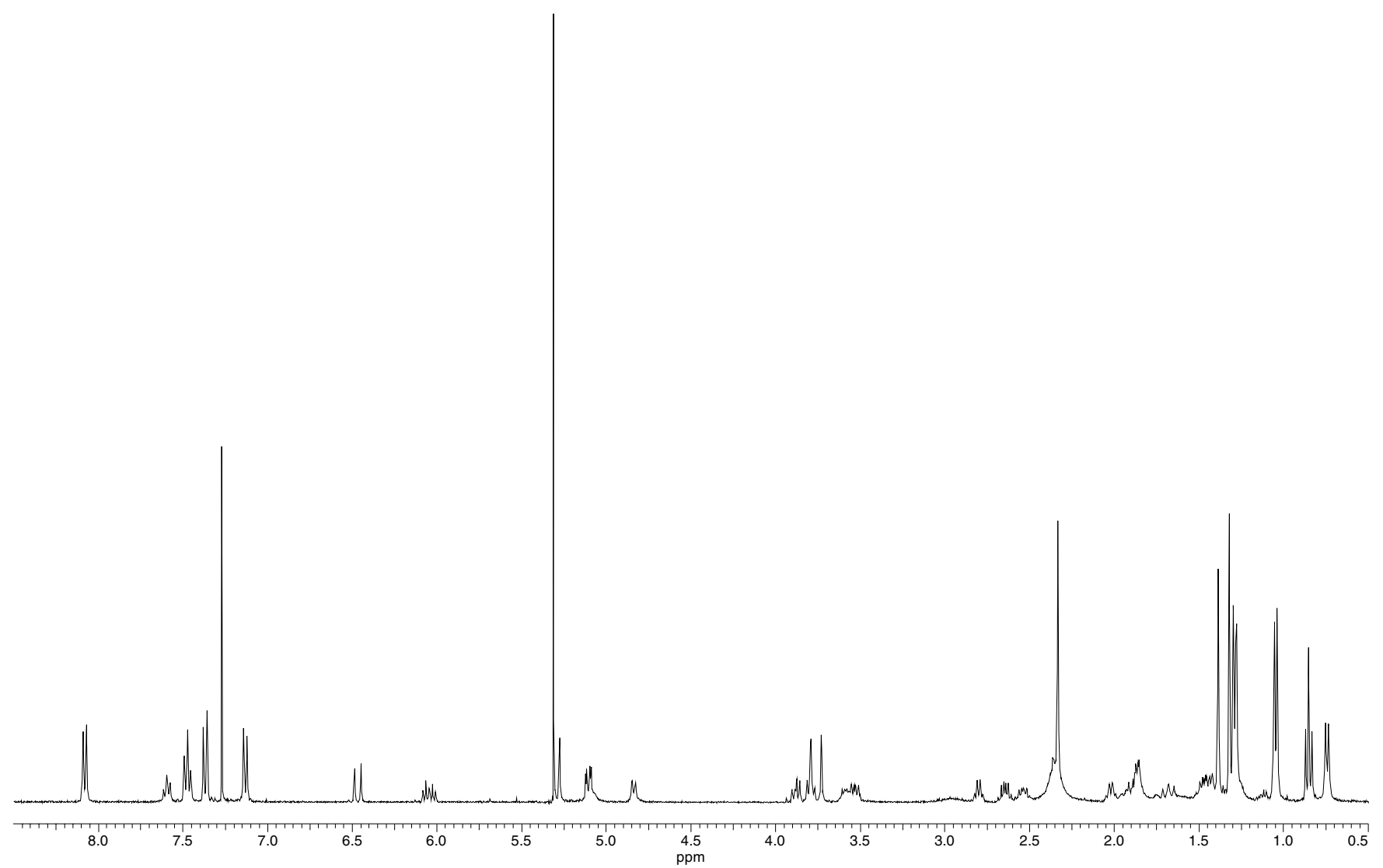

